\title{
Chemical Tracers of Pre-Brown Dwarf Cores Formed Through Turbulent Fragmentation
}

\author{
Jonathan Holdship ${ }^{1}$ and Serena Viti ${ }^{1}$ \\ ${ }^{1}$ Department of Physics and Astronomy, University College London, \\ WC1E 6BT, , United Kingdom \\ email: jrh@star.ucl.ac.uk
}

\begin{abstract}
A gas-grain time dependent chemical code, UCL_CHEM, has been used to investigate the possibility of using chemical tracers to differentiate between the possible formation mechanisms of brown dwarfs. We model the formation of a pre-brown dwarf core through turbulent fragmentation by following the depth-dependent chemistry in a molecular cloud through the step change in density associated with an isothermal shock and the subsequent freefall collapse once a bound core is produced. Trends in the fractional abundance of molecules commonly observed in star forming cores are then explored to find a diagnostic for identifying brown dwarf mass cores formed through turbulence. We find that the cores produced by our models would be bright in $\mathrm{CO}$ and $\mathrm{NH}_{3}$ but not in $\mathrm{HCO}^{+}$. This differentiates them from models using purely freefall collapse as such models produce cores that would have detectable transitions from all three molecules.
\end{abstract}

Keywords. stars: low-mass, brown dwarfs, stars: formation, astrochemistry

\section{Introduction}

A brown dwarf is a substellar object with a mass less than $0.075 \mathrm{M}_{\odot}$; the H-burning limit. Since their discovery in 1995 (Rebolo (1995)), the origin of brown dwarfs has challenged simple theories of star formation. Various theories have been put forward to explain how objects much smaller than the average Jeans mass in a molecular cloud can become gravitationally bound and unstable to collapse.

In the turbulent fragmentation theory theory, bound cores are formed by the collision of supersonic flows in molecular clouds (Padoan \& Nordlund (2002)). The resulting isothermal shocks produce dense cores of gas which may collapse to form stars. The range of core masses produced depend on the cloud's turbulent power spectrum and spans masses from the smallest brown dwarfs to the most massive stars. Both of the referenced works produce a core mass function consistent with theory and observation, implying that a universal star formation process could be responsible for all masses of stellar objects.

In this work, an adaptation of the chemical model UCL_CHEM (Viti (2004)) was produced to simulate the formation of a brown dwarf through turbulent fragmentation. The aim is to present molecular tracers that could be used to identify a pre-brown dwarf core that has formed through shock compression. Chemical abundances are highly sensitive to the cloud density so the fast increase that accompanies a shock in a molecular cloud should give rise to certain molecules having abundances incompatible with a less violent density increase such as a freefall collapse.

\section{Model}

The gas-grain chemical code UCL_CHEM was used for this work. The code follows the abundances of 214 species through a network of over 2400 reactions. Initially, solar elemental abundances are assumed and the code is run from a density of $10^{2} \mathrm{~cm}^{-3}$ to a density typical of a molecular cloud $\left(10^{4} \mathrm{~cm}^{-3}\right)$. The code models a single parcel of gas and has no geometry. A pseudo-1D model is built up by modeling 10 points spaced along a line of sight from the centre 


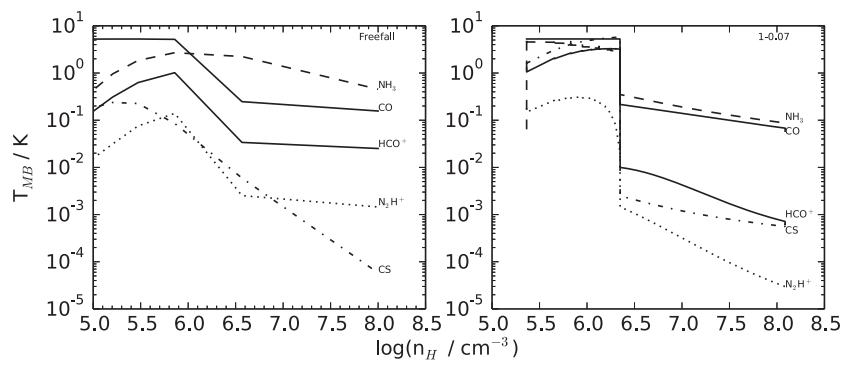

Figure 1. Brightness temperature expected for the brightest, ground observable transitions of five molecules. On the left, a freefall model is shown and on the right a turbulent fragmentation model in which a $0.07 \mathrm{M} \odot$ core is formed from a cloud.

of a core to the edge. The chemical abundances of these points are averaged to give the core average.

We model the formation of bound core as happening from the centre outwards. Colliding flows produce a dense sphere of gas which grows radially as more gas is deposited onto the core. Each of the points in the model is held at a constant preshock density and assigned a radial distance from the centre of the core. At the time when the core radius has grown to encompass a point, its density is instantaneously increased to the critical density at which a core of chosen mass would be self bound. From there, the density of a shocked point increases as the core collapses in freefall.

\section{Results}

The code outputs the fractional abundances of each molecule involved in the chemical network. We can use these to predict observables that would be seen in pre-brown dwarf cores. To do this, we used the radiative transfer code RADEX (Van der Tak (2007)) with collisional data from the LAMDA database (Schier (2005)). In this way, we were able to predict which molecules would be observable based not only on their fractional abundance but also on the expected excitation of individual transitions.

Above, the output of RADEX using our model abundances as inputs. Based on current work observing prestellar cores and bound clumps in molecular clouds, we consider everything below $0.1 \mathrm{~K}$ to be undetectable with current telescopes. Using this criteria we note two of the five most commonly observed molecular species would be observable in a pre-brown dwarf core formed through turbulent fragmentation: $\mathrm{CO}$ and NH3. In a comparable freefall model, $\mathrm{HCO}+$ is border line observable. The observation of this molecule in a pre-brown dwarf core could be used to rule out an isothermal shock.

\section{References}

Padoan, P. \& Nordlund, Å. 2002, ApJ, 576, 870

Rebolo, R., Zapatero Osorio, M. R. \& Martín, E. L. 1995, Nature, 377, 129

Schier, F.L., van der Tak, F.F.S., van Dishoeck E.F., Black, J. H. 2005, A 64 A 432, 369

Van der Tak, F.F.S., Black, J.H., Schier, F.L., Jansen, D.J., van Dishoeck, E.F., 2007, $A \mathscr{E} A$ 468,627

Viti, S., Collings, M.P., Dever, J.W., McCoustra, M.R.S. \& Williams, D. A. 2004, MNRAS, 354,1141 